\title{
Thin films of poly[(9,9-dioctylfluorene)-co-thiophene] deposited on ITO by the Langmuir-Schaefer and Langmuir-Blodgett techniques
}

\author{
Danielly Cristina Bento ${ }^{1} \cdot$ Camila Gouveia Barbosa $^{2} \cdot$ Lucas Kaique Martins Roncaselli $^{3}$. \\ Wesley Renzi ${ }^{4} \cdot$ José Leonil Duarte $^{4}$. Clarissa de Almeida Olivati ${ }^{3}$. \\ Laura Oliveira Péres ${ }^{2} \cdot$ Henrique de Santana ${ }^{1}$ (i)
}

Received: 19 September 2016/Accepted: 31 October 2016/Published online: 22 November 2016

(C) Springer Science+Business Media New York 2016

\begin{abstract}
The copolymer, poly[(9,9-dioctylfluorene)-cothiophene] (PDOF-co-Th), prepared by the Suzuki reaction, was deposited as thin films on ITO substrates using the Langmuir-Blodgett and Langmuir-Schaefer techniques. The optical properties of the films were studied by UV-Vis reflectance, photoluminescence and time-resolved photoluminescence, and the electrical properties by electrochemical impedance spectroscopy as a function of film thickness. Raman spectroscopy and atomic force microscopy (AFM) were also used with the aim of elucidating the interactions between layers and the morphology of the PDOF-co-Th thin films at the interface with the ITO. The absorption and emission spectra showed shifts and alterations in the intensities of the bands as a function of film thickness, related to the formation of aggregates and increases in the radiation selfabsorption effect. The Nyquist and Bode phase diagrams revealed that the charge transfer process at the ITO/PDOFco-Th/electrolyte interfaces was enhanced as the number of layers increased. Raman spectra of monolayer films revealed a preferential interaction between the fluorine rings and the electrode surface that was not strong enough to alter the optical and electrical properties of the copolymer. However, as shown in the AFM images of the PDOF-co-Th film
\end{abstract}

Henrique de Santana

hensan@uel.br

1 Departamento de Química, CCE, Universidade Estadual de Londrina, Londrina, PR 86051-990, Brazil

2 Laboratório de Materiais Hibridos, Universidade Federal de São Paulo, Diadema, SP, Brazil

3 Faculdade de Ciência e Tecnologia (FCT), UNESP, Presidente Prudente, SP 19060-900, Brazil

4 Departamento de Física, Universidade Estadual de Londrina, Londrina, PR 86051-990, Brazil surfaces, there is an increase in roughness and compaction that proves that the material agglomerates as the number of layers increases, suggesting that the thiophene rings also come closer together on the substrate surface, enhancing the charge transfer process at this interface.

\section{Introduction}

Several studies to determine the optical and electrical properties of the $\pi$-conjugated polymers with the aim of using these materials as active layers in solar cells, OLEDs, and electrochromic devices have been reported in the literature [1-6]. A major challenge in these studies is achieving efficient luminescence combined with high mobility, structural control and stability, all desired properties in polymeric films [5, 7]. Various techniques have been utilized to deposit conjugated homopolymers and copolymers in order to study the properties of the interfaces.

To improve optical, electrical, magnetic and other proprieties, new deposition techniques have been developed, including the Langmuir technique used to study monolayers in the aqueous subphase (Langmuir films) and the transfer of this film in a controlled and organized manner to various solid substrates, producing Langmuir-Blodgett (LB) and Langmuir-Schaeffer (LS) films. The Langmuir-Schaeffer technique is used on rigid monolayers, because transfer by the Langmuir-Blodgett technique is impaired, and only a few layers can be transferred without using phospholipids [8,9].

The many advantages provided by these techniques include the possibility of obtaining organized films with controlled thickness, which is very important if these films are to be used in optoelectronic applications, in which thickness control and reproducibility affect the efficiency of the devices [10-12]. 
Against this backdrop, the aim of this study was to evaluate the properties of poly[(9,9-dioctylfluorene)-cothiophene] (PDOF-co-Th) deposited on ITO using the Langmuir-Blodgett and Langmuir-Schaeffer techniques. Because of its high fluorescence quantum yield [7, 13], the optical properties of this material were investigated by $\mathrm{UV}-\mathrm{Vis}$, fluorescence and measurement of luminescence decay time as a function of thickness. The decay time measurements were carried out to complement the photoluminescence results. To study the electrochemical behavior of these films, the charge transfer processes were elucidated using Nyquist and Bode phase diagrams obtained by electrochemical impedance spectroscopy (EIS), applying a potential defined by Cyclic Voltammetry (CV). Raman spectroscopy was used to elucidate possible interactions between the copolymer layers and between the copolymer and the substrate. Used in conjunction with atomic force microscopy (AFM), it was possible to study the effect of copolymer compaction as a function of the number of copolymer film layers on the ITO.

\section{Experimental data}

\subsection{Copolymer films}

The poly[(9,9-dioctylfluorene)-co-thiophene] was prepared by the Suzuki reaction using $\mathrm{Pd}$ as a catalyst. The following compounds were combined in a $250-\mathrm{mL}$ threenecked round-bottom flask: $2.12 \mathrm{mmol}$ of 2,5-dibromothiophene (Aldrich, 95\%), $2.62 \mathrm{mmol}$ of 9,9-dioctylfluorene2,7-diboronic acid (Aldrich, 96\%), $120 \mathrm{~mL}$ of toluene and $10 \mathrm{~mL}$ of a $2 \mathrm{~mol} / \mathrm{L}$ potassium carbonate solution. All oxygen from the reflux system was removed by nitrogen flow and $0.0013 \mathrm{mmol}$ of tetrakis-(triphenylphosphine) palladium catalyst added. The reaction was maintained under reflux in an inert atmosphere for $72 \mathrm{~h}$ at $100^{\circ} \mathrm{C}$. After this period, $0.5 \mathrm{mmol}$ 2-bromothiophene (Aldrich, $98 \%$ ) was added and the reaction kept under the same conditions for $24 \mathrm{~h}$. Next, the reaction was cooled to room temperature. Then $10 \mathrm{~mL}$ of hydrogen peroxide was added and the solution stirred until effervescence ended. The solution was stored in a dark refrigerator ready for the next steps. The synthesized copolymer was isolated by extraction with toluene and the solvent removed by rotoevaporation. The copolymer was purified by solubilization in chloroform and recrystallization in methanol.

\subsection{Film deposition by the Langmuir-Schaefer technique}

A Langmuir-Schaefer (LS) film is made up of one or more layers of Langmuir film, and deposited by horizontal contact between the substrate and monolayer. To improve deposition, the substrate is slowly brought into contact at the interface while the Langmuir film remains stabilized. The substrate is then raised slowly and the hydrophobic end of the molecule adheres to it. The poly[(9,9dioctylfluorene)-co-thiophene] was deposited in a Langmuir trough (KSV 5000) using a $0.2 \mathrm{mg} / \mathrm{mL}$ solution of polymer in chloroform. The experiments were conducted at room temperature $\left(22^{\circ} \mathrm{C}\right)$ using a barrier speed compression of $10 \mathrm{~mm} / \mathrm{min}$. The surface pressure used for LS deposition was $30 \mathrm{mN} / \mathrm{m}$. LS films of poly[(9,9dioctylfluorene)-co-thiophene] were superimposed to obtain 1, 5 and 25 layers on an ITO substrate.

\subsection{Film deposition by the Langmuir-Blodgett (LB) technique}

The LB technique allows the deposition of one or more discrete layers. However, deposition is conducted vertically, immersing the substrate into the aqueous subphase and then removing it. In order to transfer the first monolayer, the solid support was removed (elevated) from the aqueous subphase, causing the polar portion (hydrophilic) of the molecules to interact with the solid substrate. To prepare the films, $25 \mu \mathrm{L}$ of a $5 \mathrm{mg} / \mathrm{mL}$ copolymer solution in chloroform was dripped in the aqueous subphase using a Langmuir trough (mini-model, KSV instruments). After $30 \mathrm{~min}$, the interface was compressed at a surface pressure of $30 \mathrm{mN} / \mathrm{m}$ and using a barrier speed compression of $10 \mathrm{~mm} / \mathrm{min}$. The film was transferred from the subphase to the solid substrate by dripping at $5 \mathrm{~mm} / \mathrm{min}$ and applying a constant surface pressure of $30 \mathrm{mN} / \mathrm{m}$, forming films of 1 and 5 layers. The experiments were conducted at room temperature $\left(22{ }^{\circ} \mathrm{C}\right)$.

\subsection{Characterization}

UV-Vis reflectance spectra were obtained using a UV-2600 UV-Vis spectrophotometer (Shimadzu) coupled to an ISR2600 integrating sphere. The light source consisted of two lamps, one tungsten and the other deuterium. Transmission spectra were collected in the range $\lambda=200-700 \mathrm{~nm}$.

The photoluminescence (PL) spectra were obtained using an excitation source consisting of a $1.5 \mathrm{~mW}$ diode laser emitting at $405 \mathrm{~nm}$, and recorded using a $0.5 \mathrm{~m}$ monochromator equipped with a 150 lines/mm grating, a GaAs photomultiplier and a SR510 lock-in amplifier (Stanford).

Photoluminescence decay time was measured on a FluoTime 200 (PicoQuant) using the time-correlated single photon counting (TCSPC) technique. The FluoTime 200 was equipped with a MCP detector and 405-nm pulsed laser $(0.03-80 \mathrm{MHz})$. Measurements were taken through 
$0.5 \mathrm{~mm}$ slits and the laser emission was also recorded as an IRF for later use in adjusting the decay. The system's time resolution was $50 \mathrm{ps}$ and the detection range from 300 to $800 \mathrm{~nm}$. For these studies, the laser pulse frequency was set to $10 \mathrm{MHz}$.

Raman spectra were obtained on a DeltaNu Advantage $532^{\circledR}$ portable Raman spectrometer set to $532 \mathrm{~nm}$ and $8 \mathrm{~cm}^{-1}$ resolution. DeltaNu NuSpec software with baseline resources was used to remove background fluorescence.

Impedance diagrams at various potentials were obtained using an Autolab PGSTAT $302 \mathrm{~N}$ potentiostat coupled to a microcomputer running NOVA 1.8 , with a FRAM32 impedance module, varying the frequency from $100 \mathrm{kHz}$ to $0.01 \mathrm{~Hz}$. Measurements were initiated after waiting for $5 \mathrm{~min}$, the time required to reach the stationary state at which the current is constant. Potentials were determined by reference to $\mathrm{Ag} / \mathrm{AgCl}$ in a Luggin capillary in a $0.100 \mathrm{~mol} \mathrm{~L}{ }^{-1}$ solution of $\mathrm{LiClO}_{4}$ in acetonitrile $\left(\mathrm{LiClO}_{4} /\right.$ $\mathrm{ACN})$.

Atomic force microscopy (AFM) was performed on NanoSurf FlexAFM (Liestal-Switzerland) equipment. The analyses were carried out in air and images obtained in intermittent contact mode, using silicon pins (PP-NCLR50). Scanning was set to free oscillation frequency and various amplitudes, depending on the stability and contrast obtained. The setpoint was set to $30-50 \%$ of free oscillation amplitude.

\section{Results and discussion}

Figure 1 shows the absorption and emission spectra obtained for the PDOF-co-Th copolymer films as a function of the number of layers deposited by the LangmuirBlodgett (LB, Fig. 1a) and Langmuir-Schaefer (LS, Fig. 1b) techniques.

Figure 1a shows the absorption and emission spectra of the pure copolymer in a chloroform solution and films consisting of 1,5 and 9 discrete layers. Comparing the results obtained for the pure copolymer in solution and in the solid state, there is a red shift in the absorption and emission spectra. In addition, there is a new absorption maximum at $462 \mathrm{~nm}$. In spite of these differences, the spectra profiles are well-defined. The UV-Vis spectrum of the monolayer film deposited on ITO by the LS technique (Fig. 1b) does not show a well-defined absorption band. On the other hand, the spectra of the films with 5 and 25 layers show an absorption maximum at $435 \mathrm{~nm}$ and a shoulder at $459 \mathrm{~nm}$.
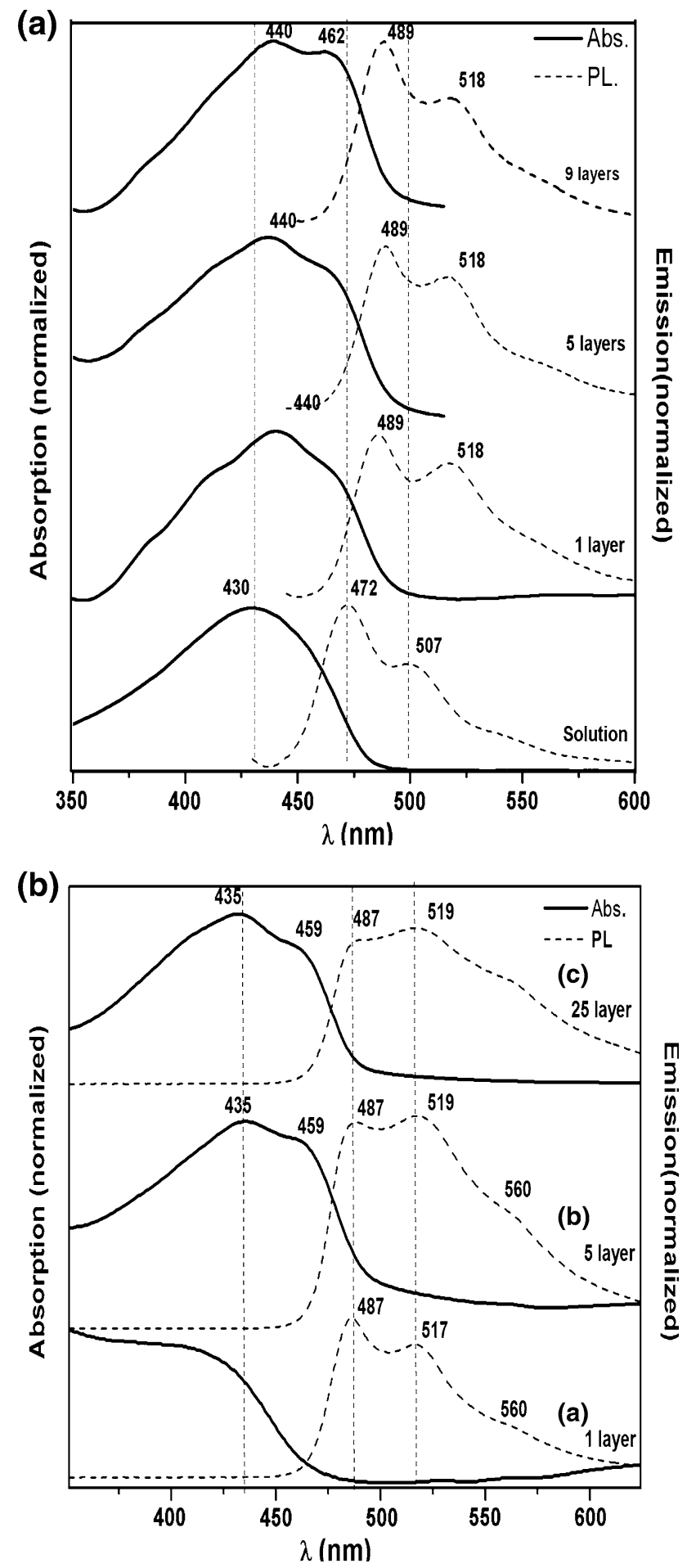

Fig. 1 Poli[(9,9-dioctylfluorene)-co-thiophene] spectra: a Absorption and emission (excitation at maximum absorption) in solution $\left(5.6 \times 10^{-6} \mathrm{~g} / \mathrm{mL}\right.$ in chloroform) and in films of 1,5 and 9 discrete layers deposited by the Langmuir-Blodgett (LB) technique; b absorption and emission (excitation at $430 \mathrm{~nm}$ ) in films deposited by the Langmuir-Schaefer (LS) technique of 1; 5 and 25 layers 
As seen Fig. 1a (spectrum of the pure copolymer dissolved in chloroform), the wide absorption band centered on $430 \mathrm{~nm}$ is related to the absorption of the PDOF-co-Th copolymer [13]. One possible explanation for the shift and change observed in the films prepared by the LB and LS techniques is related to the presence of aggregates in these samples, characterized by changes in the absorption and emission spectra, which can be vibronically resolved [14].

The formation of aggregates originated by inter-chain interactions occurs typically in concentrated solid state systems and impairs the photo- and electroluminescence of the conjugated polymers by enhancing the non-radiative deactivation processes of the polymer chain. When the formation of these structures occurs, the absorption and emission spectra are shifted to the lower-energy red region [14-16].

The formation of shoulders in the absorption spectra at 462 and $459 \mathrm{~nm}$ indicates the formation of aggregates, since there is absorption by more than one species in the solid state. In the films, the compression and the consequent packing of the molecules may boost inter-chain interactions leading to the formation of polymer aggregates, causing shifts in the absorption and emission spectra.

In the emission spectra, the maxima obtained by the two techniques are almost identical (487 and 517-521 nm and a shoulder at $560 \mathrm{~nm}$ ). There were no significant differences in the emissions of the films, even for the LS monolayer film, which merely showed more clearly defined bands. Nevertheless, there was a small red shift in these spectra compared to the copolymer in solution (Fig. 1a). This red shift is related to the packing of the molecules inside the films.

Band gap values were calculated for the polymers deposited by the two techniques, using the slope of the absorption band $[17,18]$. In the films deposited by LS, gap energy values were $2.60 \mathrm{eV}$ (monolayer), $2.44 \mathrm{eV}$ (5 layers) and $2.48 \mathrm{eV}$ (25 layers). The values obtained for the LB films were $2.57 \mathrm{eV}$ (copolymer in solution), $2.53 \mathrm{eV}$ (monolayer), $2.52 \mathrm{eV}$ (5 layers) and $2.52 \mathrm{eV}$ (9 layers). The same band gap value for the copolymer in solution has already been reported in the literature [13].

The results obtained show that the monolayer film prepared by LS has a band gap value similar to that of the polymer in solution. This also shows that the material deposited does not suffer the effects of interactions between neighboring polymeric chains (through the formation of aggregates and excimers) and that the interaction between the polymer film and the substrate is not strong enough to change the optical properties of the copolymer. This is not the case for the monolayer LB film. It is interesting to observe that all LB film band gap values were very similar and largely unaffected by the number of layers deposited.
Variations in the emission intensities of the vibronic progressions according to the number of layers in the films were also observed in all photoluminescence spectra. There was a difference in intensity ratio between the $0-0$ and $0-1$ vibronic peaks at $487 \mathrm{~nm}(2.54 \mathrm{eV})$ and $517-521 \mathrm{~nm}$ $(2.39-2.37 \mathrm{eV})$. In the monolayer film, the $0-0$ peak was more intense than the $0-1$ peak, and in the 5-layer film these intensities were the same. In the 25-layer film, the 0-1 peak was more intense. The higher intensity of the $0-1$ transition, involving phonon creation, suggests that the film's self-absorption increases with film thickness [19].

Tables 1, 2, 3, 4 show the emission decay times obtained for films deposited by LS and LB. To compare the results obtained for each technique, we used the same detection wavelength $\left(\lambda_{\mathrm{em}}\right)$ for all films. Table 1 shows the results obtained for emission decay times from three emission peaks for the LS films.

Since the decay results for higher energy peaks are dependent on the equipment response time ( $\sim 70 \mathrm{ps})$ and can be even lower, mean decay times (as a function of intensity) were also analyzed. The results of the adjustments are shown in Table 2.

The results obtained show a drop in decay time $(\tau)$ as the number of layers increases. This corroborates the idea that increasing the thickness of the films causes them films to change from acting as isolated chains (solution/thin film) to agglomerates (thick film), characterized by the predominance of faster emissions.

Furthermore, along with the idea that a thick film is formed once 5 layers has been reached, it is possible to verify that higher energy emissions remain almost unchanged in terms of decay time at thicknesses of 5-25 layers. The effect of increasing the number of layers is conspicuous in the intensity of the shadow at $560 \mathrm{~nm}$. The increase in emission intensity could be associated with the self-absorption of the film, i.e. the lower energy peaks (486 and $518 \mathrm{~nm}$ ) remain less intense due to the internal filter effect.

The position of the shadow at $560 \mathrm{~nm}$, together with the elevated decay times ( $\sim 2 \mathrm{~ns})$, are characteristics associated with isolated thiophene chains, as suggested in studies on P3HT [20]. We can therefore assume that this emission is due to the thiophene component of the copolymer.

Based on a comparison with the decay times in Tables 1 and 2, the change of deposition technique (Tables 3, 4) suggests the formation of thinner films, in which the molecules are more isolated, resulting in a higher emission decay time.

With the aim of checking whether there is any interaction between the copolymer layers and whether the copolymer is interacting with the substrate, experiments were carried out using IES, Raman spectroscopy and the AFM morphology results. 
Table 1 Emission decay times of films deposited by the LS technique at excitation of $405 \mathrm{~nm}$

\begin{tabular}{|c|c|c|c|c|c|c|c|c|c|}
\hline \multirow[t]{2}{*}{ Peak } & \multicolumn{3}{|c|}{1 Layer } & \multicolumn{3}{|c|}{5 Layers } & \multicolumn{3}{|c|}{25 Layers } \\
\hline & $\tau(\mathrm{ns})$ & $\Delta \tau(\mathrm{ns})$ & $\%$ & $\tau(\mathrm{ns})$ & $\Delta \tau(\mathrm{ns})$ & $\%$ & $\tau(\mathrm{ns})$ & $\Delta \tau(\mathrm{ns})$ & $\%$ \\
\hline \multirow[t]{2}{*}{$486 \mathrm{~nm}$} & 0.050 & 0.001 & 90 & 0.033 & 0.001 & 86 & 0.042 & 0.001 & 96 \\
\hline & 0.28 & 0.01 & 10 & 0.17 & 0.01 & 14 & 0.39 & 0.04 & 4 \\
\hline \multirow[t]{2}{*}{$518 \mathrm{~nm}$} & 0.071 & 0.001 & 85 & 0.050 & 0.001 & 88 & 0.052 & 0.001 & 92 \\
\hline & 0.74 & 0.03 & 15 & 0.70 & 0.04 & 12 & 0.69 & 0.04 & 8 \\
\hline \multirow[t]{2}{*}{$563 \mathrm{~nm}$} & 0.128 & 0.001 & 62 & 0.109 & 0.001 & 62 & 0.085 & 0.001 & 74 \\
\hline & 1.57 & 0.05 & 38 & 1.58 & 0.06 & 38 & 1.45 & 0.07 & 26 \\
\hline
\end{tabular}

Table 2 Mean higher energy emission decay times of films deposited by the LS technique

\begin{tabular}{llll}
\hline Peak & $\begin{array}{l}\text { 1 Layer } \\
\tau(\mathrm{ns})\end{array}$ & $\begin{array}{l}\text { 5 Layers } \\
\tau(\mathrm{ns})\end{array}$ & $\begin{array}{l}25 \text { Layers } \\
\tau(\mathrm{ns})\end{array}$ \\
\hline $486 \mathrm{~nm}$ & 0.073 & 0.054 & 0.055 \\
$518 \mathrm{~nm}$ & 0.170 & 0.129 & 0.105 \\
\hline
\end{tabular}

Table 3 Emission decay times of films deposited by the LB technique at excitation of $405 \mathrm{~nm}$

\begin{tabular}{llllllll}
\hline Peak & \multicolumn{1}{l}{1 Layer } & \multicolumn{5}{l}{5 Layers } \\
\cline { 2 - 3 } \cline { 6 - 7 } & $\tau(\mathrm{ns})$ & $\Delta \tau(\mathrm{ns})$ & $\%$ & & $\tau(\mathrm{ns})$ & $\Delta \tau(\mathrm{ns})$ & $\%$ \\
\hline $486 \mathrm{~nm}$ & 0.075 & 0.001 & 90 & 0.048 & 0.001 & 86 \\
& 0.56 & 0.03 & 10 & 0.59 & 0.03 & 14 \\
$518 \mathrm{~nm}$ & 0.092 & 0.001 & 82 & 0.071 & 0.001 & 78 \\
& 0.74 & 0.03 & 18 & 0.75 & 0.03 & 22 \\
$563 \mathrm{~nm}$ & 0.17 & 0.01 & 67 & 0.18 & 0.01 & 60 \\
& 1.94 & 0.07 & 33 & 2.11 & 0.08 & 40 \\
\hline
\end{tabular}

Table 4 Mean higher energy emission decay times of films deposited by the LB technique

\begin{tabular}{lll}
\hline Peak & $\begin{array}{l}\text { 1 Layer } \\
\tau(\mathrm{ns})\end{array}$ & $\begin{array}{l}\text { 5 Layers } \\
\tau \text { (ns) }\end{array}$ \\
\hline $486 \mathrm{~nm}$ & 0.089 & 0.092 \\
$518 \mathrm{~nm}$ & 0.190 & 0.179 \\
\hline
\end{tabular}

Figure 2 shows the Nyquist and Bode phase diagrams obtained at $0.100 \mathrm{~mol} \mathrm{~L}^{-1} \mathrm{LiClO}_{4} / \mathrm{ACN}$ when a potential of $1.00 \mathrm{~V}$ is applied to films of PDOF-co-Th deposited in discrete layers on ITO using the LS technique. Inserts were required in Fig. 2a to show the highest frequencies in the enlarged Nyquist diagram.

In the Nyquist diagrams (Fig. 2a and insert), the formation of two imperfect semicircles at high frequencies is observed for PDOF-co-Th deposited on ITO in 5 and 25 layers. The imperfection in the semicircles is probably due to the non-homogeneous nature of the material's surface. Thus, these semicircles relate to the process of charge transfer resistance at the $\mathrm{PDOF}-\mathrm{co}-\mathrm{Th} / \mathrm{ClO}_{4}{ }^{-}$and ITO/ PDOF-co-Th interfaces $[21,22] . \mathrm{R}_{\mathrm{CT}}$ values at the ITO/
PDOF-co-Th interface were $3.39 \times 10^{3} \Omega(5$ layers $)$ and $3.16 \times 10^{2} \Omega$ (25 layers). For the monolayer film of PDOF-co-Th, only one imperfect semicircle was observed relating to the charge transfer process at the $\mathrm{PDOF-co-Th/}$ $\mathrm{ClO}_{4}{ }^{-}$interface, indicating that, for this film, the ITO/ PDOF-co-Th system is passive, i.e. the charge transfer resistance is high at this interface [3, 21-23].

The Bode phase diagrams in Fig. $2 b$ show that, for a film deposited as a monolayer, there is the formation of a non-defined, fairly resistant phase with a phase angle of close to $86^{\circ}$ at a frequency of $0.19 \mathrm{~Hz}$, which is steeper than the clearly defined phases of the 5- and 25-layer films, with a phase angle of $70^{\circ}$ (5 layers) and $36^{\circ}-64^{\circ}$ (25 layers) at respective frequencies of 2.90 and $641.5-0.12 \mathrm{~Hz}$. It can be seen that the process that is clearly defined in the 25-layer film at a frequency of $641.5 \mathrm{~Hz}$ and with a lower phase angle $\left(36^{\circ}\right)$ is more favorable than the other processes [3, 23, 24].

Figure 3 shows the Raman spectra of the copolymer films deposited on ITO in discrete layers and compared to the spectrum of the solid copolymer obtained by chemical synthesis, with the aim of verifying the nature of the deposited films.

The Raman spectrum of the copolymer as prepared in solid form (Fig. $3 \mathrm{~A}(\mathrm{~d})$ ) shows the main frequencies at 1450 (thiophene ring) and $1606 \mathrm{~cm}^{-1}$ (fluorene ring), characteristic of symmetrical stretching of the thiophene and fluorene rings $[1,25]$. The width of these bands, compared to the same frequencies observed in the spectra of the thin films (Fig. 3A(a-c)) and Fig. 3B(a,b), is narrower and the shifts observed in these bands were not very significant compared to the bands for the solid sample. However, the band at $1573 \mathrm{~cm}^{-1}$ in the solid sample spectrum, associated with symmetric stretching of the fluorene ring, was observed in the spectra of samples deposited as a monolayer using the LS and LB techniques, with two respective frequencies at $1556 \mathrm{~cm}^{-1}$ (LS) and 1569/1572 $\mathrm{cm}^{-1}$ (LB). To explain this behavior, there must be a preferential interaction between the fluorene rings and the electrode surface, responsible for the appearance of two frequencies close together.

As already reported in in situ Raman experiments [13], in which the potential was applied under same the 


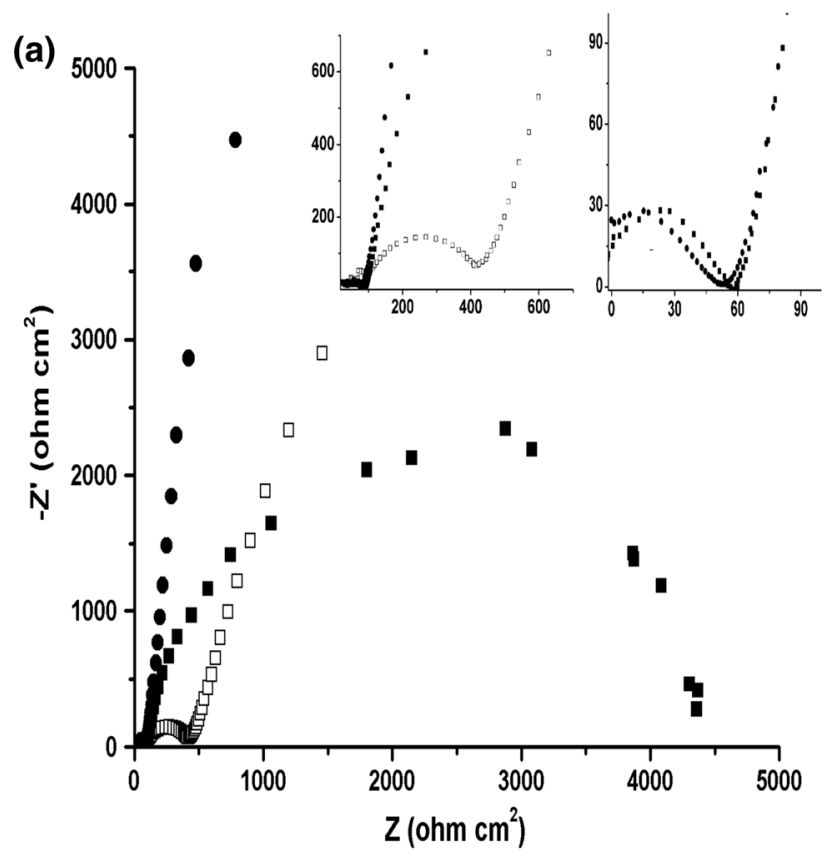

(b)

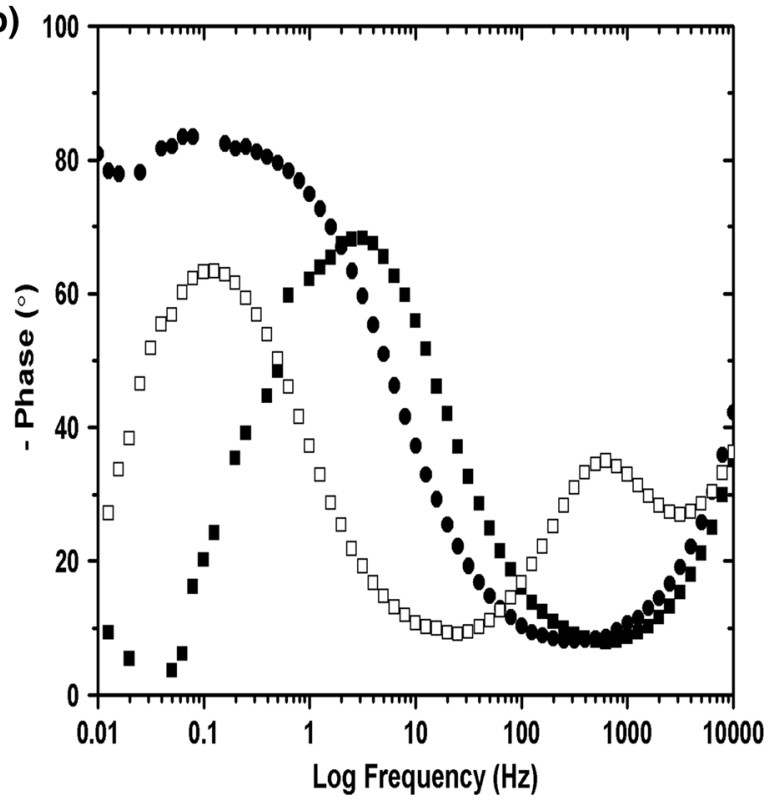

Fig. 2 Nyquist diagram (a) and bode phase diagram (b) obtained at $1.00 \mathrm{~V}$ for PDOF-co-Th films in $0.100 \mathrm{~mol} \mathrm{~L}^{-1} \mathrm{LiClO}_{4} / \mathrm{ACN}$ deposited on ITO (filledcircle) monolayer; (filledsquare) 5 layers; (opensquare) 25 layers

conditions as those used for obtaining the EIS in the present study, it was observed that the intensity of the band at $1446 \mathrm{~cm}^{-1}$ suddenly dropped and a new band at $1474 \mathrm{~cm}^{-1}$ was simultaneously observed. This behavior was characterized by the formation of the radical cation in the thiophene ring. It should also be borne in mind that the ex situ Raman results for the monolayer indicate that the fluorine rings are apparently closer to the surface of the ITO. When the number of layers is increased, it is possible
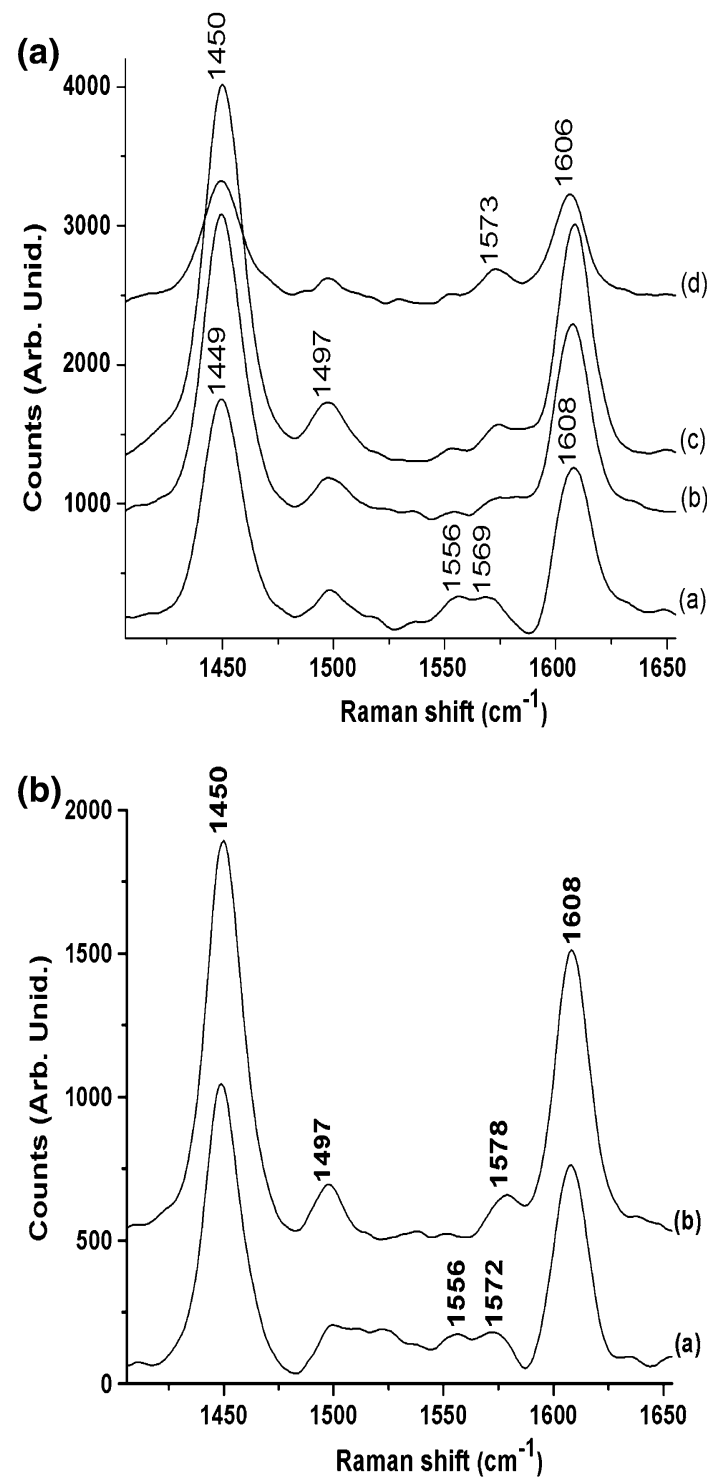

Fig. 3 Raman spectra (excitation at $532 \mathrm{~nm}$ ) of poly[(9,9-dioctylfluorene)-co-thiophene] films deposited by (a) the Langmuir-Schaefer (LS) technique in $(a) 1$; $(b) 5$ and $(c) 25$ layers and $(d)$ in solid form as prepared, and (b) by the Langmuir-Blodgett (LB) technique, in (a) 1 and $(b) 5$ layers

that the chains are further compacted. This compacting must be bringing the thiophene rings closer to the surface. The IES results indicate that, at the ITO/PDOF-co-Th interface, the thiophene rings are responsible for the charge transfer process.

To study the morphology of the PDOF-co-Th films deposited on ITO in discrete layers, atomic force microscopy (AFM) was used to obtain topographical images of the materials. Figure 4 shows 3D topographical images of the various films deposited by LS.

The AFM images of the surface of films deposited using the LS technique show the formation of grains that agglomerate under the material's surface in a non- 
homogeneous form and that significantly increase in number as a function of the number of layers deposited on the ITO. To study these morphological changes, the rootmean-square (RMS) roughness [26, 27] was determined for the total area and for less aggregated (A) and more aggregated (B) regions of each film, as shown in Table 5.

The RMS values show that for the monolayer PDOF-coTh film (Fig. 4a) surface roughness varies slightly

(a)

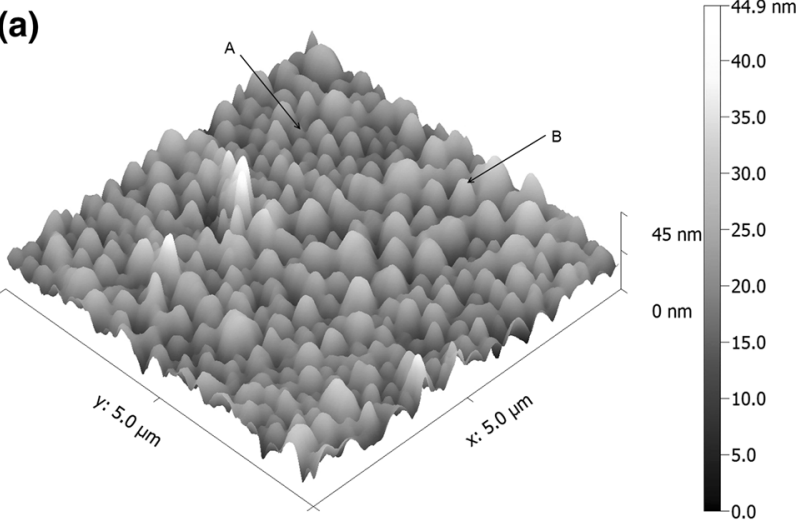

(b)

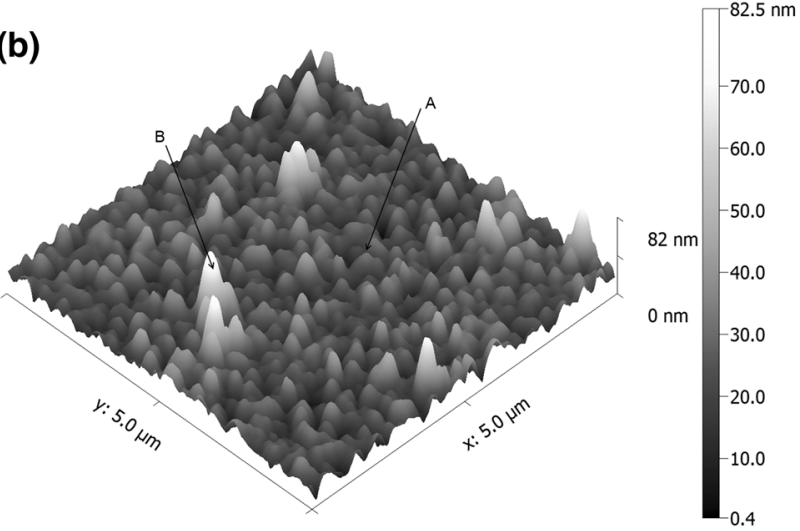

(c)

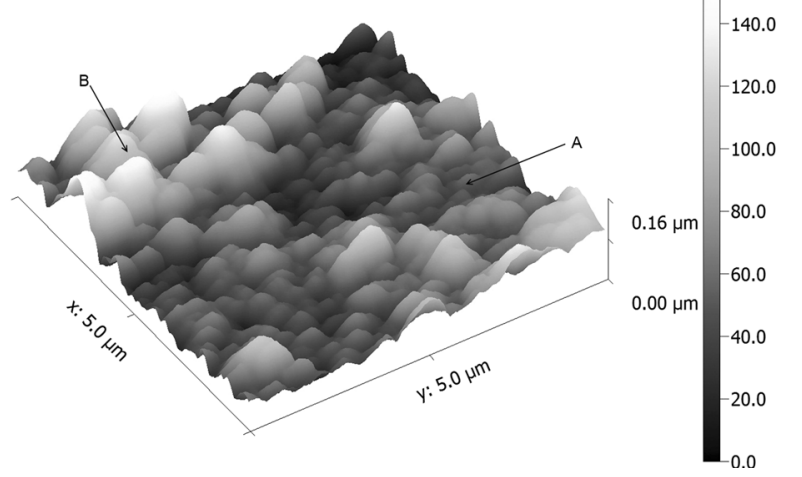

Fig. 4 AFM 3D topographical images $(5.00 \mu \mathrm{m} \times 5.00 \mu \mathrm{m})$ of PDOF-co-Th deposited on ITO by the Langmuir-Schaefer (LS) technique: a monolayer; b 5 layers; c 25 layers. (A) least aggregated regions and $(B)$ most aggregated regions
Table 5 Roughness values obtained from AFM images (Fig. 5) of PDOF-co-Th films deposited on ITO by the LS technique in 1, 5 and 25 layers

\begin{tabular}{lll}
\hline Samples & Area & RMS roughness (nm) \\
\hline PDOF-co-Th 1 layer & Total & 5.00 \\
& A & 4.21 \\
PDOF-co-Th 5 layers & B & 5.41 \\
& Total & 7.78 \\
& A & 6.01 \\
PDOF-co-Th 25 layers & B & 12.76 \\
& Total & 25.61 \\
& A & 13.38 \\
& B & 38.88 \\
\hline
\end{tabular}

(a)

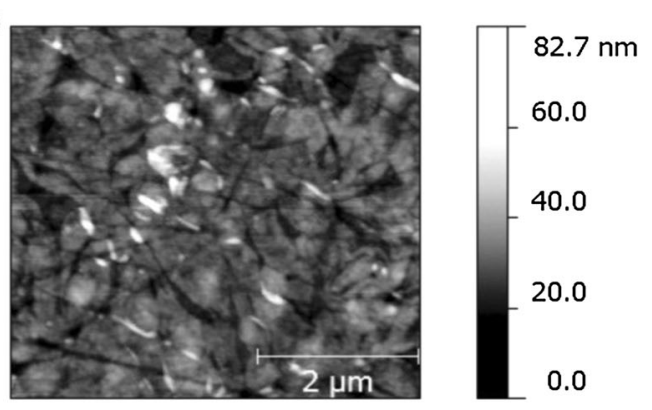

(b)

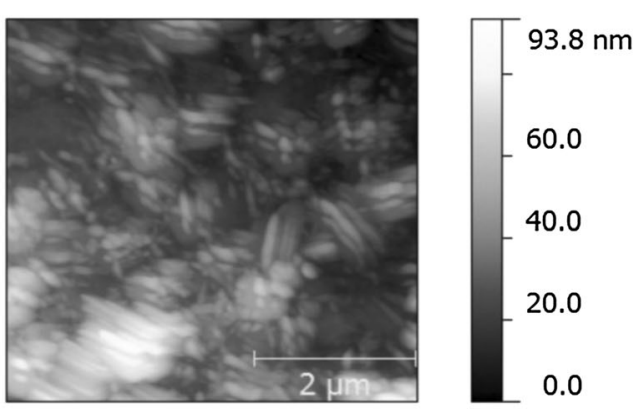

(c)

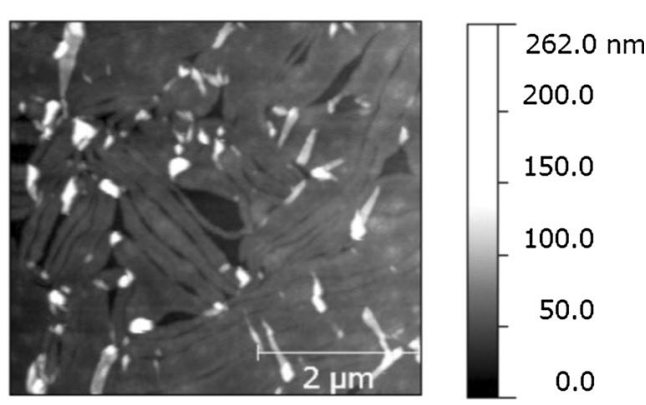

Fig. 5 AFM topographical images $(5.00 \mu \mathrm{m} \times 5.00 \mu \mathrm{m})$ of PDOFco-Th films deposited on ITO by the Langmuir-Blodgett (LB) technique: a 1 layer; $\mathbf{b} 5$ layers; c multiple layers

compared to the total area RMS with areas A and B, characterizing the surface of this film as homogeneous. However, the RMS of the 5- and 25-layer PDOF-co-Th 
films exhibits a greater difference when the total area RMS is compared to areas A and B. Furthermore, there was an increase in MSR as the number of layers increased.

Figure 5 shows the topographical images of the various PDOF-co-Th films deposited by the LB technique.

The surface images of the PDOF-co-Th films deposited on ITO using the LB technique (Fig. 5), in contrast to the formation of "grains" observed in the images of the films deposited using the LS technique, showed the formation of rod-shaped aggregates. In Fig. 5a, the monolayer film's surface appears amorphous and exhibits a homogeneous surface with low roughness. Interestingly, the surface image of the 5-layer PDOF-co-Th film appears more organized as rod shapes, in addition to displaying darker areas, possibly formed as a result of higher agglomeration. This organized structuring is more visible on the multilayer film's surface (Fig. 5c), showing the formation of spaghetti-like structures. The multilayer film surface also shows a drop in the number of white regions (surface peaks) compared to the monolayer film. This characteristic means that the spaghetti-like structures are more regular in size and give the film a more compact surface.

The increase in roughness (Fig. 4) and compaction (Fig. 5) observed in the AFM surface images of the PDOFco-Th films is evidence of the agglomeration of the material as the number of layers is increased, and suggests that the thiophene rings are coming closer to the surface, enhancing the charge transfer process (Fig. 2) and the predominance of faster emissions (Table 1) observed in the 5- and 25-layer PDOF-co-Th systems.

\section{Conclusions}

The various thin films of PDOF-co-Th copolymer deposited on ITO electrodes using the Langmuir-Blodgett and Langmuir-Schaefer techniques vary in their optical properties. Shifts in the absorption and emission spectra towards the lower-energy red region and the variation in the emission intensity of the PDOF-co-Th film indicate the formation of aggregates and an increase in the self-absorption effect as a function of thickness. This aggregation was also verified by the time-resolved photoluminescence result, showing a drop in the films' decay time $(\tau)$, i.e. a predominance of faster emissions as the number of layers increases.

The EIS spectra of the films deposited by the LangmuirSchaefer technique show a drop in $\mathrm{R}_{\mathrm{CT}}$ as a function of thickness. The charge transfer processes at the ITO/PDOFco-Th/electrolyte interface were stronger in the 5- and 25-layer films, whereas this process in the monolayer film was passive, with high charge transfer resistance at the interface.
The AFM topographical images of the film surfaces show how the agglomeration and structuring of the copolymer is dependent on the number of layers in the film on ITO. Taken in conjunction with the Raman data, we can assume that the thiophene rings are brought closer to the substrate surface by compaction, thereby enhancing the charge transfer processes, crucial to these materials in optoelectronic applications.

The effect on emission evidenced by the shadow at $560 \mathrm{~nm}$, together with the elevated decay times ( $2 \mathrm{~ns})$, were associated with the copolymer's thiophene component.

Acknowledgements We would like to express our appreciation to the Spectroscopy Laboratory (SPEC) at the PROPPG/UEL Multiuser Center. This study was supported by $\mathrm{CNPq}$ program/special visiting researcher (PVE), Process No. 401109/2014-3 and Project No. 441689/2014-0.

\section{References}

1. H. Chen, X.R. Cai, Z.G. Xu, T. Zhang, B. Song, Y. Li, Q. Jiang, M.G. Xie, Polym. Bull. 60, 581 (2008)

2. A. Bagui, S.S.K. Iyer, Org. Electron. 15, 1387 (2014)

3. D.C. Bento, E.C.R. Maia, T.N.M. Cervantes, C.A. Olivati, G. Louarn, H. De Santana, J. Mater. Sci. 26, 149 (2015)

4. W. Zhou, C. Yu, H. Chen, T. Jia, W. Zhang, G. Yu, F. Li, J. Phys. Chem. C 120, 4824 (2016)

5. X. Liu, H. Chen, S. Tan, Renew. Sustain. Energy Rev. 52, 1527 (2015)

6. C.B. Nielsen, S. Holliday, H.Y. Chen, S.J. Cryer, I. McCulloch, Acc. Chem. Res. 48, 2803 (2015)

7. L.O. Péres, S.H. Wang, J. Wéry, G. Froyer, E. Faulques, Mater. Sci. Eng. C 29, 372 (2009)

8. T.C.F. Santos, L.O. Péres, S.H. Wang, O.N. Oliveira Jr., L. Caseli, Langmuir 26, 5869 (2010)

9. A. Sakai, S.H. Wang, L.O. Peres, L. Caseli, Synth. Met. 161, 1753 (2011)

10. J. Xu, Y. Yang, J. Yu, Y. Jiang, Appl. Surf. Sci. 255, 4329 (2009)

11. A.P. Wu, M. Jikei, M.A. Kakimoto, Y. Imai, S. Ukishima, Y. Takahashi, Mol. Cryst. Liq. Cryst. A 267, 441 (1995)

12. R. Ravindranath, P.K. Ajikumar, R.C. Advincula, W. Knoll, S. Valiyaveettil, Langmuir 22, 9002 (2006)

13. C.G. Barbosa, D.C. Bento, L.O. Péres, G. Louarn, H. de Santana, J. Mater. Sci. 27, 10259 (2016)

14. Y.F. Huang, Y.J. Shiu, J.H. Hsu, S.H. Lin, A.C. Su, K.Y. Peng, S.A. Chen, W.S. Fann, J. Phys. Chem. C 111, 5533 (2007)

15. B. Valeur, Molecular Fluorescence: Principles and Applications, 1st edn. (Wiley, Paris, 2001), pp. 34-37

16. M. Vacha, J. Ha, Y. Ito, T. Shimada, J.M. Son, H. Sato, J. Appl. Phys. 97, 023514 (2005)

17. S.H. Wu, H.M. Huang, K.C. Chen, C.W. Hu, C.C. Hsu, R.C.C. Tsiang, Adv. Funct. Mater. 16, 1959 (2006)

18. Z. Peng, M.E. Galvin, Chem. Mater. 10, 1785 (1998)

19. C.M. Martin, S. Guba, M. Chandrasekhar, H.R. Chandarsekhar, R. Guentner, P.S. de Freitas, U. Scherf, Phys. Rev. B 68, 115203 (2003)

20. P.Y. Chen, A. Rassamesard, H.L. Chen, S.A. Chen, Macromolecules 46, 5657 (2013)

21. J.R. MacDonald, Electrochim. Acta 35, 1483 (1990) 
22. Y. Li, J. Gao, G. Yu, Y. Cao, A.J. Heeger, Chem. Phys. Lett. 287, 83 (1998)

23. D.C. Bento, E.C.R. Maia, P.R.P. Rodrigues, G. Louarn, H. de Santana, J. Mater. Sci. 24, 4732 (2013)

24. H. Ding, Z. Pan, L. Pigani, R. Seeber, C. Zanardi, Electrochim. Acta 46, 2721 (2001)
25. Y. Wang, Y. Liu, W. Zhu, Dyes Pigm. 113, 312 (2015)

26. A. Roigé, M. Campoy-Quiles, J.O. Ossó, M.I. Alonso, L.F. Vega, M. Garriga, Synth. Met. 161, 2570 (2012)

27. P. Alessio, M.L. Braunger, R.F. Aroca, C.A. Olivati, C.J.L. Constantino, J. Phys. Chem. C 119, 12055 (2015) 